\title{
Análise de Características Empreendedoras Utilizando Sistemas Baseados em Regras Fuzzy ${ }^{1}$
}

D.P. BELLUCCI ${ }^{2}$, Centro de Matemática, Computação e Cognição, UFABC, 09210-170 Santo André, SP, Brasil.

A.J.V. BRANDÃO ${ }^{3}$, Universidade Federal de São Carlos - UFSCar, Campus Sorocaba, 18052-780 Sorocaba, SP, Brasil.

Resumo. Neste trabalho apresentaremos um modelo que classifica pessoas quanto à sua aptidão para o empreendedorismo. Dado o caráter subjetivo deste tipo de classificação, utilizaremos no modelo uma ferramenta matemática chamada Sistema Baseado em Regras Fuzzy. A montagem das regras será feita a partir de dados do Sebrae e ao final apresentaremos um programa no qual o usuário entra com seus dados e tem como saída um diagnóstico sobre sua aptidão para o empreendedorismo.

Palavras-chave. Sistema Baseado em Regras Fuzzy, Matlab, Empreendedorismo.

\section{Introdução}

Abrir uma empresa requer algumas competências pessoais que podem fazer diferença para que o negócio tenha sucesso. É preciso que algumas características estejam presentes, como os conhecimentos, as habilidades e atitudes empreendedoras. São características decisivas para quem pretende entrar no mundo dos negócios. Um dos fatores importantes para a sobrevivência e desenvolvimento das empresas é o espírito empreendedor que, com características bem desenvolvidas, farão com que a pessoa tenha mais condições de viabilizar a empresa. [5]

Para a análise que iremos fazer, utilizaremos a Teoria Fuzzy, a qual teve início com a introdução do conceito de conjunto fuzzy por L. A. Zadeh, em 1965 [6], e que vem ganhando destaque desde então, devido a grande aplicabilidade de sua teoria. Em um conjunto clássico, um elemento pertence ou não pertence a tal conjunto. Nos conjuntos fuzzy, os elementos possuem um grau de pertinência relativo a seu conjunto, definidos pela função de pertinência. O mesmo vale quando tratamos da Lógica Fuzzy. Na Lógica Clássica, trabalhamos com proposições verdadeiras ou falsas. Já na Lógica Fuzzy, as proposições são imprecisas devido ao fato de existirem incertezas em suas variáveis. É por esse motivo que trabalharemos com Lógica Fuzzy para a avaliação que faremos a seguir, pois analisar as características de um certo indivíduo é certamente de caráter subjetivo.

\footnotetext{
${ }^{1}$ Agradecemos à CAPES pelo suporte financeiro.

${ }^{2}$ danilobellucci@gmail.com

3 adilsonvb@ufscar.br
} 
Um Sistema Baseado em Regras Fuzzy (SBRF) é um sistema que tenta reproduzir a estratégia de um controlador humano na resolução de problemas. Torna-se assim possível traduzir termos linguísticos, constantemente empregados por especialistas com o intuito de controlar suas tarefas, em fórmulas matemáticas, possibilitando a automação de certas atividades [1]. Iremos utilizar um SBRF para classificarmos pessoas quanto a sua aptidão para ser um empreendedor [2]. Para tanto, usaremos o Fuzzy Logic Toolbox do software Matlab ${ }^{\circledR}$.

Em [3] o autor também estuda um modelo de empreendedorismo utilizando teoria fuzzy, mas através de uma abordagem distinta da nossa. Apesar de elencar dez características empreendedoras, o referido autor reduz para três conjuntos de características na modelagem do problema, enquanto no nosso, trabalhamos com oito características. Outra diferença é o número de valores linguísticos utilizados pelo autor, três valores: baixo, médio e alto. Em nosso trabalho utilizamos dois valores: ausente e presente. Diante disso em [3] o autor faz uso de 19 regras e em nosso trabalho utilizamos 256 regras. Além do mais, ele utiliza o método de inferência de Takagi-Sugeno, enquanto nós utilizamos o método de inferência de Mandami.

Na próxima seção, apresentamos as principais definições sobre conjuntos e sobre lógica fuzzy. Na seção 3, definimos o que é um Sistema Baseado em Regras Fuzzy e os principais resultados referentes à esse assunto. Por fim, na seção 4, apresentamos nosso principal resultado: avaliação empreendedora utilizando SBRF. Algumas simulações podem ser vistas nesta seção final.

\section{Definições}

Nesta seção iremos apresentar alguns resultados sobre Teoria Fuzzy que nos serão úteis. Mais resultados podem ser encontrados em [1] e [4].

\subsection{Conjuntos Fuzzy}

Definição 2.1 (Conjunto Fuzzy). Seja $U$ um conjunto universo clássico. Um conjunto fuzzy é um par $\left(F, \varphi_{F}\right), \operatorname{com} F \subset U$ e $\varphi_{F}: U \rightarrow[0,1]$ é a chamada função grau de pertinência.

A imagem de um elemento de $F$ ser 0 significa que esse elemento não está no conjunto fuzzy, 1 significa que ele está completamente no conjunto, e se a imagem estiver entre 0 e 1 , caracteriza o grau de pertinência do elemento no conjunto.

Definição 2.2. A união de $A$ e $B$ é o subconjunto fuzzy $A \cup B$ de $U$ cuja função de pertinência é dada por

$$
\varphi_{A \cup B}(x)=\max _{x \in U}\left\{\varphi_{A}(x), \varphi_{B}(x)\right\} .
$$

Definição 2.3. A intersecção entre $A$ e $B$ é o subconjunto fuzzy $A \cap B$ de $U$ cuja função de pertinência é dada por

$$
\varphi_{A \cap B}(x)=\min _{x \in U}\left\{\varphi_{A}(x), \varphi_{B}(x)\right\} .
$$


Definição 2.4. O complementar de A em relação a U é o subconjunto $A^{\prime}$ de $\mathrm{U}$ cuja função de pertinência é dada por

$$
\varphi_{A^{\prime}}(x)=1-\varphi_{A}(x), x \in U
$$

\subsection{Lógica Fuzzy}

Em lógica fuzzy utilizamos os operadores $\Delta$ (t-norma), $\nabla$ (t-conorma) e $\Rightarrow$ para modelar os conectivos lógicos $e$, ou e então, respectivamente. A t-norma que utilizaremos neste trabalho é o operador $\Delta(x, y)=\min \{x, y\}=x \wedge y$. A t-conorma será o operador $\nabla_{1}(x, y)=\max \{x, y\}$.

Definição 2.5. Uma variável linguística $X$ no universo $U$ é uma variável cujos valores assumidos por ela são subconjuntos fuzzy de $U$.

Definição 2.6. Uma relação fuzzy $\boldsymbol{R}$ sobre $U_{1} \times U_{2} \times \cdots \times U_{n}$ é qualquer subconjunto fuzzy de $U_{1} \times U_{2} \times \cdots \times U_{n}$. Assim, uma relação fuzzy $\boldsymbol{R}$ é definida por uma função de pertinência $\varphi_{\boldsymbol{R}}: U_{1} \times U_{2} \times \cdots \times U_{n} \rightarrow[0,1]$.

Definição 2.7. O produto cartesiano fuzzy dos subconjuntos fuzzy $A_{1}, A_{2}, \ldots, A_{n}$ de $U_{1}, U_{2}, \ldots, U_{n}$, respectivamente, é a relação fuzzy $A_{1} \times A_{2} \times \cdots \times A_{n}$, cuja função de pertinência é dada por

$$
\varphi_{A_{1} \times A_{2} \times \cdots \times A_{n}}\left(x_{1}, x_{2}, \ldots, x_{n}\right)=\varphi_{A_{1}}\left(x_{1}\right) \wedge \varphi_{A_{2}}\left(x_{2}\right) \wedge \cdots \wedge \varphi_{A_{n}}\left(x_{n}\right),
$$

onde $\wedge$ representa o mínimo.

Definição 2.8. Considere $R$ e $S$ duas relações fuzzy binárias em $U \times V$ e $V \times W$, respectivamente. A composição $R \circ S$ é uma relação fuzzy binária em $U \times W$ cuja função de pertinência é dada por

$$
\varphi_{R \circ S}(u, w)=\sup \left\{\min \left\{\varphi_{R}(u, v), \varphi_{S}(v, w)\right\}\right\}
$$

\section{Sistemas Baseados em Regras Fuzzy}

Nesta seção apresentamos alguns resultados sobre Sistemas Baseados em Regras Fuzzy. Maiores informações podem ser encontrados em [1], [2] e [4].

Um Sistema Baseado em Regras Fuzzy (SBRF) é um sistema que se utiliza da lógica fuzzy para produzir saídas para cada entrada fuzzy.

Um caso particular de um SBRF é o chamado Controladores Fuzzy, que é aquele em que tentamos reproduzir a estratégia de um controlador humano. No controlador fuzzy, a entrada representa uma "condição", e a saída representa uma "ação".

\subsection{Controlador Fuzzy}

Um controlador fuzzy é composto pelos módulos de fuzzificação, de base de regras, de inferência fuzzy e de defuzzificação, como podemos ver no esquema. Explicaremos, a seguir, o que desempenha cada módulo de um Controlador Fuzzy. 


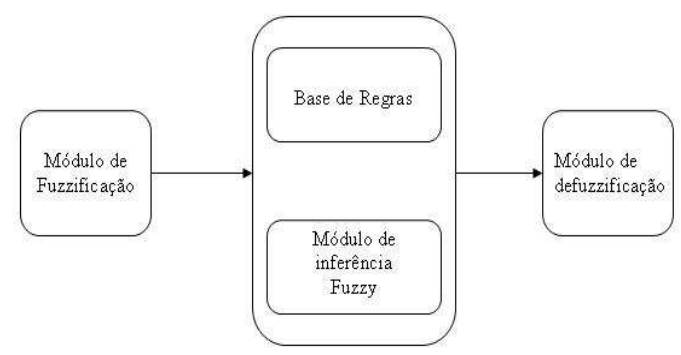

Figura 1: Esquema geral de um controlador fuzzy.

- Módulo de fuzzificação: nesta etapa, as entradas do sistema são modeladas por conjuntos fuzzy, isto é, associa-se a entrada à uma função de pertinência. Mesmo que a entrada seja crisp (clássica), essa será fuzzificada por meio de sua função característica.

- Módulo da base de regras: a base de regras é compostas por proposições do tipo

$$
\begin{gathered}
\text { Se } x_{1} \text { é } A_{1} \text { e } x_{2} \text { é } A_{2} \text { e } \ldots \text { e } x_{n} \text { é } A_{n} \text { então } \\
u_{1} \text { é } B_{1} \text { e } u_{2} \text { é } B_{2} \text { e } \ldots \text { e } u_{m} \text { é } B_{m},
\end{gathered}
$$

onde as variáveis linguísticas são modeladas por conjuntos fuzzy (funções de pertinência). Quando dizemos que $x_{i}$ é $A_{i}$ queremos dizer que a pertinência de $x_{i}$ em $A_{i}$ está entre 0 e 1 .

Os conjuntos fuzzy que compõem o estado $\left(A_{i}\right)$ são chamados de antecedentes, e os que compões a resposta $\left(B_{i}\right)$ são chamados consequentes.

- Módulo de inferência fuzzy: aqui definem-se quais serão os conectivos lógicos usados para estabelecer a relação fuzzy que modela a base de regras. O sucesso do sistema fuzzy depende deste módulo, já que ele fornecerá a saída a ser adotada pelo controlador a partir de cada entrada. O método de inferência usa as t-normas e t-conormas para traduzir matematicamente as sentenças da base de regras, que são ligadas pelos conectivos e e ou. Pela sua simplicidade, iremos utilizar o método de inferência de Mamdani, o qual é definido a seguir.

1. em cada regra $R_{j}$, da base de regras fuzzy, a condicional "se x é $A_{i}$ então u é $B_{i}$ " é modelada pela aplicação $\wedge$ (mínimo);

2. adota-se a t-norma $\wedge$ para o conectivo lógico $\mathbf{e}$;

3. para o conectivo lógico ou adota-se a t-conorma $\vee$ (máximo) que conecta as regras fuzzy da base de regras.

Formalmente, a relação fuzzy $\mathbf{R}$ (que é a relação que modela a base de regras) é o subconjunto fuzzy de $X \times U$, cuja função de pertinência é dada por

$$
\varphi_{\mathbf{R}}(x, u)=\max _{1 \leq i \leq r}\left(\varphi_{R_{i}}(x, u)\right)=\max _{1 \leq i \leq r}\left[\varphi_{A_{i}}(x) \wedge \varphi_{B_{i}}(u)\right],
$$


no qual $x$ representa o estado e $u$ representa o controle, $r$ é o número de regras que compõem a base de regras, e $A_{i}$ e $B_{i}$ são os subconjuntos fuzzy da regra $i$. Cada um dos valores $\varphi_{A_{i}}(x)$ e $\varphi_{B_{i}}(u)$ é interpretado como o grau com que $x$ e $u$ estão nos subconjuntos fuzzy $A_{i}$ e $B_{i}$, respectivamente.

- Módulo de Defuzzificação: no controlador fuzzy, a cada entrada fuzzy, o módulo de inferência produz uma saída fuzzy que indica o controle a ser adotado. O defuzzificador converte tal saída fuzzy em um número real. Adotaremos um método específico de defuzzificação, chamado centro de gravidade, também conhecido como centróide ou centro de área. Esse método nos dá a média das áreas de todas as figuras que representam os graus de pertinência de um subconjunto fuzzy.

\section{Empreendedorismo}

Para a análise que iremos fazer, utilizaremos um SBRF afim de classificarmos pessoas quanto a sua aptidão para ser um empreendedor.

Para avaliarmos se uma pessoa está apta a se tornar empreendedora ou não, iremos estudar as características a seguir, com seus respectivos conceitos e média nacional. Tais características foram extraídas de [5].

1. Planejamento Formal: planejamento formal corresponde ao quanto o empreendedor busca formalizar procedimentos e planejar as atividades do seu negócio. Média Nacional: 8,0.

2. Desafio: é o desejo e motivação em superar limites e concorrentes, buscando ser sempre o melhor naquilo que faz. Média Nacional: 7,9.

3. Inovação: representa a criatividade e espírito de inovação do empreendedor. Média Nacional: 7,4 .

4. Competência Estratégica: indica sua capacidade e confiança para articular recursos e pessoas em direção a um objetivo profissional e de negócios maior. Média Nacional: 8,8.

5. Risco: corresponde a disposição e aceitação de riscos calculados na sua vida profissional. Média Nacional: 8,8.

6. Relacionamento: corresponde ao carisma ao lidar com colegas de trabalho, funcionários, clientes e fornecedores. Média Nacional: 8,4.

7. Dedicação: corresponde à disposição do empreendedor se dedicar e abrir mão da vida pessoal pelo sucesso nos negócios. Média Nacional: 6,8.

8. Pensamento Analítico: O Pensamento Analítico é a capacidade de avaliar contextos de forma holística, usando a lógica e a razão ao abordar um problema. Média Nacional: 8,4. 


\subsection{Modelagem}

Para realizarmos nosso trabalho, precisaremos construir uma base de regras. Neste caso, serão 256 regras. O antecedente será constituído pelas 8 entradas: planejamento formal, desafio, inovação, competência estratégica, risco, relacionamento, dedicação e pensamento analítico; e o consequente terá 1 saída: aptidão ao empreendedorismo, que irá assumir os valores apto, possibilidade ou inapto.

Cada entrada possui uma "nota de corte", segundo a média brasileira fornecida por [5]. Tais notas serão modeladas por conjuntos fuzzy. Se a entrada for maior ou igual a nota de corte (com uma certa tolerância), então tal característica será entendida como presente, e ausente caso contrário. Como exemplo, a figura a seguir mostra a função de pertinência para a característica "inovação". Todas as características serão fuzzificadas através de uma função de pertinência que avalia se tal será considerada presente ou ausente, de acordo com a média nacional.

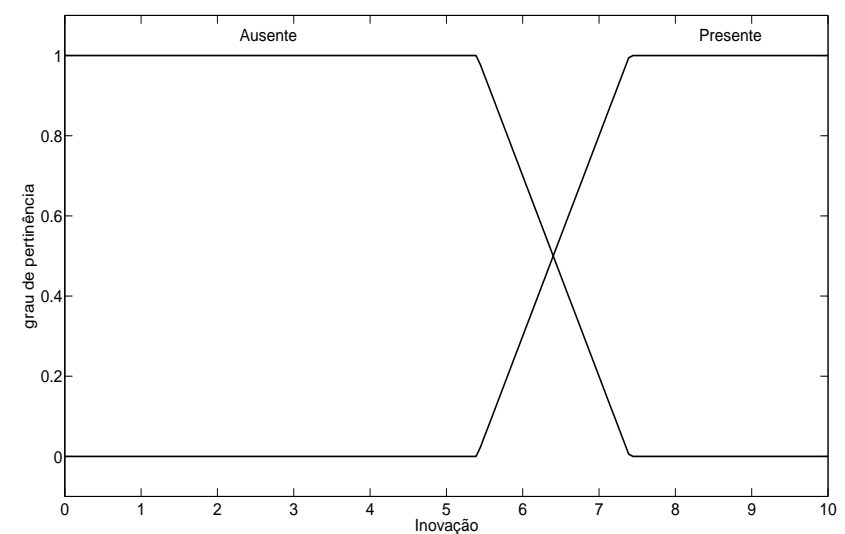

Figura 2: Função de pertinência para a característica "inovação".

As regras foram construídas com a ajuda de um consultor do Sebrae. Algumas destas regras estão ilustradas na Tabela 1.

\begin{tabular}{|c|c|c|c|c|c|c|c|c|}
\hline $\begin{array}{l}\text { P. Formal } \\
\text { Ausente }\end{array}$ & $\begin{array}{l}\text { Desafio } \\
\text { Ausente }\end{array}$ & $\begin{array}{c}\text { Inov. } \\
\text { Ausente }\end{array}$ & $\begin{array}{l}\text { Comp. Estrat. } \\
\text { Ausente }\end{array}$ & $\begin{array}{c}\text { Risco } \\
\text { Ausente }\end{array}$ & $\begin{array}{c}\text { Relac. } \\
\text { Ausente }\end{array}$ & $\begin{array}{l}\text { Dedic. } \\
\text { Ausente }\end{array}$ & $\begin{array}{l}\text { P. Anal. } \\
\text { Ausente }\end{array}$ & $\begin{array}{c}\text { Aptidão } \\
\text { Inapto }\end{array}$ \\
\hline Ausente & Ausente & A usente & Ausente & Ausente & Ausente & Ausente & Presente & Inapto \\
\hline$\cdot$ & . & . & . & . & & & & \\
\hline Presente & Presente & Ausente & Presente & Ausente & Ausente & Presente & Ausente & Possib. \\
\hline Presente & Presente & Ausente & Presente & Ausente & Ausente & Presente & Presente & Possib. \\
\hline $\begin{array}{l}\text { Presente } \\
\text { Presente }\end{array}$ & $\begin{array}{l}\text { Presente } \\
\text { Presente }\end{array}$ & $\begin{array}{l}\text { Presente } \\
\text { Presente }\end{array}$ & $\begin{array}{l}\text { Presente } \\
\text { Presente }\end{array}$ & $\begin{array}{l}\text { Presente } \\
\text { Presente }\end{array}$ & $\begin{array}{l}\text { Presente } \\
\text { Presente }\end{array}$ & $\begin{array}{l}\text { Presente } \\
\text { Presente }\end{array}$ & $\begin{array}{l}\text { Ausente } \\
\text { Presente }\end{array}$ & $\begin{array}{l}\text { Apto } \\
\text { Apto }\end{array}$ \\
\hline
\end{tabular}

Tabela 1: Base de Regras: empreendedorismo.

Usaremos o Fuzzy Logic Toolbox do software Matlab ${ }^{\circledR}$, que processa os módulos de um Sistema Baseado em Regras Fuzzy. O método de inferência que utilizamos foi o Método de Mamdani, e o método de defuzificação é o centro de gravidade. 
A tabela abaixo mostra o resultado de algumas pessoas que procuraram o Sebrae/PA entre os meses de março e abril de 2009, afim de iniciarem seus próprios negócios. Segundo nosso programa, os resultados dessas pessoas foram:

\begin{tabular}{|c|c|c|c|c|c|c|c|c|}
\hline Planej. Formal & $\begin{array}{l}\text { Desafio } \\
7.5\end{array}$ & Inovação & Comp. Estrat. & $\begin{array}{l}\mathrm{Risco} \\
8\end{array}$ & $\begin{array}{l}\text { Relac. } \\
9.5\end{array}$ & $\begin{array}{l}\text { Dedic. } \\
7.5\end{array}$ & Pens. Anal. & $\begin{array}{l}\text { Resp. } \\
\text { Possib. }\end{array}$ \\
\hline 6 & 7.5 & 6.5 & 8 & 7 & 8.5 & 7.5 & 8 & Possib. \\
\hline 8 & 9 & 7 & 8 & 8.5 & 8 & 9 & 9 & Apto \\
\hline 9 & 9 & 8.5 & 9 & 8 & 8.5 & 9 & 8 & Apto \\
\hline 6 & 7 & 6.5 & 6 & 7.5 & 6 & 7 & 6 & Inapto \\
\hline 9 & 7.5 & 8.5 & 8 & 7 & 7.5 & 8 & 8.5 & Possib. \\
\hline 6 & 6.5 & 7 & 7 & 6.5 & 7.5 & 8 & 7.5 & Inapto \\
\hline 8 & 8 & 9 & 9.5 & 9 & 8.5 & 8 & 7.5 & Apto \\
\hline 6 & 8 & 8 & 6.5 & 8 & 6.5 & 7 & 7.5 & Possib. \\
\hline 8 & 7.5 & 8.5 & 8 & 9 & 8.5 & 7.5 & 8 & Apto \\
\hline 6 & 6.5 & 6.5 & 6 & 7 & 8 & 7.5 & 8 & Possib. \\
\hline
\end{tabular}

A figura 3 mostra a interface do programa feito no Matlab para facilitar a pesquisa. Nele, basta entrarmos com os valores obtidos na pesquisa para obtermos o resultado.

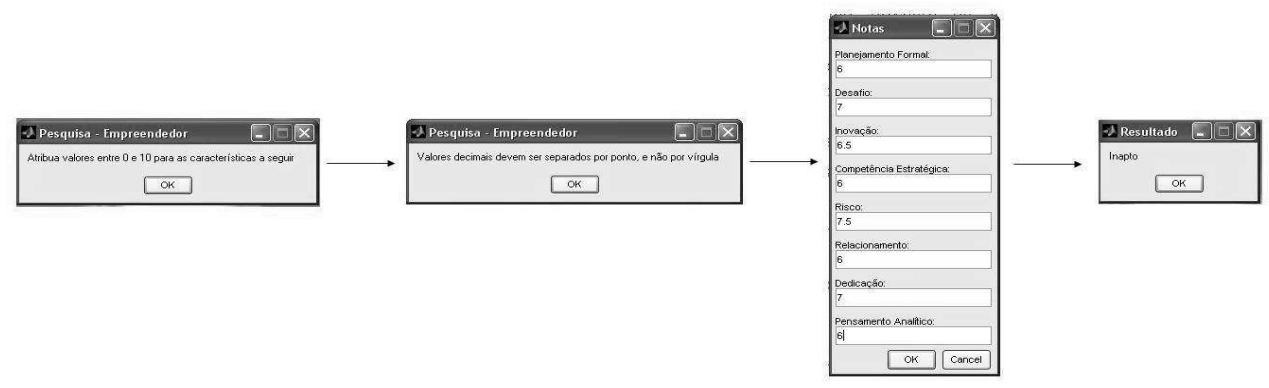

Figura 3: Interface do programa feito no Matlab para avaliação empreendedora.

\section{Conclusão}

Ao analisarmos o perfil empreendedor de uma pessoa, por meio de pesquisa, um indivíduo poderá detectar se tem condições para ser empreendedor e em qual característica deverá desenvolver mais o seu potencial.

A análise destas características é subjetiva e daí a pertinência de se modelar matematicamente tais problemas via teoria dos conjuntos fuzzy. Neste trabalho utilizamos um sistema baseado em regras fuzzy no qual, a partir da avaliação de cada característica relevante de empreendedorismo, obtivemos uma avaliação da aptidão do potencial empreendedor.

É importante salientar que a validação do modelo não é imediata. A pesquisa foi feita com pessoas que pretendem abrir uma empresa, ou seja, poderemos fazer a validação do modelo depois de um certo tempo que a empresa foi aberta, avaliando se a empresa obteve sucesso ou não, o qual poderá ser feito num trabalho futuro. 


\title{
Agradecimentos
}

Agradecemos aos pareceristas anônimos pelas sugestões que, em muito, contribuíram para a versão final deste artigo.

\begin{abstract}
In this work we present a model which will qualify people according to their entrepreneurship skills. Given the subjective nature of this type of classification, the model will be based on a tool called Fuzzy Rule-Based System. The construction of the rules will be made using data of Sebrae and, at the end, we will introduce a program where the user inserts various information and the program gives out as a result the diagnosis regarding the entrepreneurship skills.
\end{abstract}

Keywords. Fuzzy Rule-Based System, Matlab, Entrepreneurship.

\section{Referências}

[1] L.C. Barros, R.C. Bassanezi, "Tópicos de Lógica Fuzzy e Biomatemática", Coleção IMECC - Textos Didáticos, vol. 5, IMECC-UNICAMP, Campinas/SP, 2006 .

[2] D.P. Bellucci, "Sistemas Baseados em Regras Fuzzy e Aplicações", Dissertação de Mestrado, UFABC, 2009.

[3] P.D.P. Carneiro, "Metodologia Fuzzy Aplicada para Predição do Comportamento Empreendedor", Dissertação de Mestrado, Ibmec, 2008.

[4] W. Pedrycz, F. Gomide, "Fuzzy Systems Engineering: Toward Human-Centric Computing", IEEE, Wiley-Interscience, 2007.

[5] M.R. Veit, C.G. Filho, "O Perfil do Potencial Empreendedor e o Impacto no Desempenho do Negócio", Dissertação de Mestrado, FUMEC, 2006.

[6] L.A. Zadeh, "Fuzzy Sets, Fuzzy Logic, and Fuzzy Systems: Selected Papers by Lotfi A. Zadeh", World Scientific Publishing Company, 1996. 